\title{
Dietary menhaden oil: effects on the rate and magnitude of modification of phospholipid fatty acid composition of mouse heart and brain
}

\author{
BY JOY E. SWANSON, J. MARK BLACK AND JOHN E. KINSELLA \\ Lipid Research Laboratory, Institute of Food Science, Cornell University, Ithaca, \\ New York 14853, USA
}

(Received 28 July 1987 - Accepted 26 January 1988)

1. Male CD-1 white mice, $18-20 \mathrm{~g}$ body-weight, were given semi-purified diets containing $100 \mathrm{~g}$ menhaden oil (MO) or hydrogenated coconut oil ( $\mathrm{HCO}) / \mathrm{kg}$ for $23 \mathrm{~d}$. Mice were killed on days $0,3,5,7,14,23$. After $23 \mathrm{~d}$ of MO supplementation the remaining mice were switched to the $\mathrm{HCO}$ diet for an additional $10 \mathrm{~d}$.

2. The progressive change(s) in the polyunsaturated fatty acid (PUFA) composition of cardiac and brain phospholipid classes were followed during the MO supplementation and depletion periods.

3. The content of fatty acids $20: 5 n-3,22: 5 n-3$ and $22: 6 n-3$ increased immediately following ingestion of the MO diet and continued to increase at a steady rate in both heart and brain phospholipid classes.

4. In general, the period required to reach steady-state was 1 week for $n-3$ PUFA and 18:2n-6, and 2 weeks for $20: 4 n-6$.

5. Cessation of MO consumption for $10 \mathrm{~d}$ resulted in marked decreases in the content of $n-3$ PUFA and increases in $n$-6 PUFA in cardiac phospholipids in particular. Brain phospholipids were less responsive.

6. The results suggest that dietary fish oil must be consumed for at least 1 week before maximum changes in PUFA composition are observed, and fish oil ingestion must be continuous to maintain elevated $n-3$ PUFA levels in heart and brain phospholipids.

Dietary fish oils exert many beneficial physiological effects (Lands, 1986; Simopoulos et al. 1986) which have led to considerable interest in determining the quantitative relation between dietary intake of fish oils and tissue $n-3$ polyunsaturated fatty acid (PUFA) content. However, there are few reports which address the rates and extent of incorporation and distribution of $n-3$ PUFA in various tissues in man and experimental animals following fish oil ingestion (Thorngren \& Gustafson, 1981; Iritani \& Narita, 1984; Herold \& Kinsella, 1986; Swanson et al. 1987a). Differences in the magnitude of modification of tissue fatty acid composition and alteration in eicosanoid synthesis induced by fish oil ingestion have also been observed between species, tissues and lipid classes (Croft et al. 1984; Lokesh et al. 1985; Sanders, 1985a, b; Knapp et al. 1986).

The consumption of dietary fish oils, seafood or purified $n-3$ PUFA by man or animals results in the incorporation of eicosapentaenoate $(20: 5 n-3)$ and docosahexaenoate $(22: 6 n-3)$ with a concomitant reduction of linoleate $(18: 2 n-6)$ and arachidonate (20:4n-6) in tissue lipids (Bruckner et al. 1984; Herold \& Kinsella, 1986; Swanson \& Kinsella, 1986). These changes in fatty acid composition resulted in alterations in the ratio, thromboxane $\mathrm{A}_{2}\left(\mathrm{TXA}_{2}\right)$ :prostacyclin $\mathrm{I}_{2}$ which may be important in ameliorating coronary arterial disease (Lokesh et al. 1985; Sanders, 1985 b; Herold \& Kinsella, 1986). The specific activity of membrane-bound enzymes can also be changed following alteration of membrane fatty acid composition (Flier et al. 1985; Swanson et al. 1987b).

Therefore, it is imperative to know not only the magnitude of $n-3$ PUFA incorporation into various tissues but also the rate of incorporation and depletion, duration of feeding and dose required to reach a steady-state of $n-3$ PUFA in tissue lipids. This information is needed in order to ascertain the extent to which fish oil consumption may affect different biochemical processes such as eicosanoid synthesis or enzyme activity. In addition, 
Table 1. Fatty acid composition (wt \%) of dietary lipids as given to mice

\begin{tabular}{lcc}
\hline \hline Fatty acid & $\begin{array}{c}\text { Hydrogenated } \\
\text { coconut oil }\end{array}$ & $\begin{array}{c}\text { Menhaden } \\
\text { oil }\end{array}$ \\
\hline $12: 0$ & $41 \cdot 25$ & $0 \cdot 27$ \\
$14: 0$ & $16 \cdot 49$ & $8 \cdot 24$ \\
$16: 0$ & $9 \cdot 90$ & $15 \cdot 31$ \\
$16: 1 n-7$ & - & $11 \cdot 76$ \\
$18: 0$ & $12 \cdot 32$ & $4 \cdot 74$ \\
$18: 1 n-9$ & $3 \cdot 37$ & $14 \cdot 73$ \\
$18: 2 n-6$ & - & $17 \cdot 66$ \\
$18: 3 n-3$ & - & $2 \cdot 42$ \\
$18: 4$ & - & $3 \cdot 33$ \\
$22: 1 n-11$ & - & $0 \cdot 22$ \\
$20: 4 n-6$ & - & $0 \cdot 47$ \\
$20: 5 n-3$ & - & $12 \cdot 66$ \\
$22: 5 n-6$ & - & $1 \cdot 06$ \\
$22: 5 n-3$ & $22: 6 n-3$ & $4 \cdot 50$ \\
\hline \hline
\end{tabular}

knowledge of the kinetics of fish-oil-induced fatty acid modification should be valuable in the design of clinical studies to determine the effectiveness of dietary fish oils on various diseases, and also help in the development of an accurate method of assessing patient compliance in dietary intervention trials.

The object of the present study was to determine the rate of incorporation, the period required to reach steady-state and the rate of depletion of specific PUFA in mouse heart and brain phospholipids $(\mathrm{PL})$ following consumption of dietary menhaden oil.

\section{MATERIALS AND METHODS}

\section{Animals}

Male CD-1 white mice (Charles River, CASDBR, Wilmington, MA), 18-20 g body-weight, were given a commercial ration (Prolab RMH 1000; Agway Inc., Syracuse, NY) for 1 week. The mice were then randomly assigned to one of two dietary regimens, a menhaden oil (MO)-based diet or a hydrogenated-coconut-oil (HCO)-based diet, and housed, eight mice per cage. Food and water were provided ad lib. and a $12 \mathrm{~h}$ dark- $12 \mathrm{~h}$ light cycle was maintained in the room. The duration of the dietary MO supplementation was $23 \mathrm{~d}$ after which the remaining mice were switched to the HCO diet.

\section{Diets}

The diet ingredients (g/ $\mathrm{kg}$ diet) included: glucose 500, casein 240, AIN-76 mineral mix (US Biochemicals, Cleveland, OH) 22, AIN-76 vitamin mix (US Biochemicals) 60, methionine 3 , cellulose 55 , butylated hydroxytoluene $0 \cdot 1$, zinc carbonate $0 \cdot 1$, all-rac- $\alpha$-tocopheryl acetate $(600 \mu \mathrm{g} / \mathrm{g}$; Hoffman-LaRoche Inc., Nutley, NJ) $5 \mu \mathrm{g}$, safflower oil 20, plus menhaden oil (Zapata Haynie, Reedsville, VA) 100 or hydrogenated coconut oil 100. Unused food was discarded and fresh diet was given daily. Fresh diet was stored in sealed containers, flushed with nitrogen and stored at $4^{\circ}$ to minimize lipid peroxidation. Exposure of diets to air at room temperature resulted in a $2 \%$ increase in thiobarbituric-acid-reactive substances (TBA) relative to the initial values (MO, 58.5 and $\mathrm{HCO} 2.11 \mathrm{nmol} \mathrm{TBA} / \mathrm{g}$ diet) as measured by the method of Buege \& Aust (1978). The fatty acid composition of the dietary oils is shown in Table 1. 

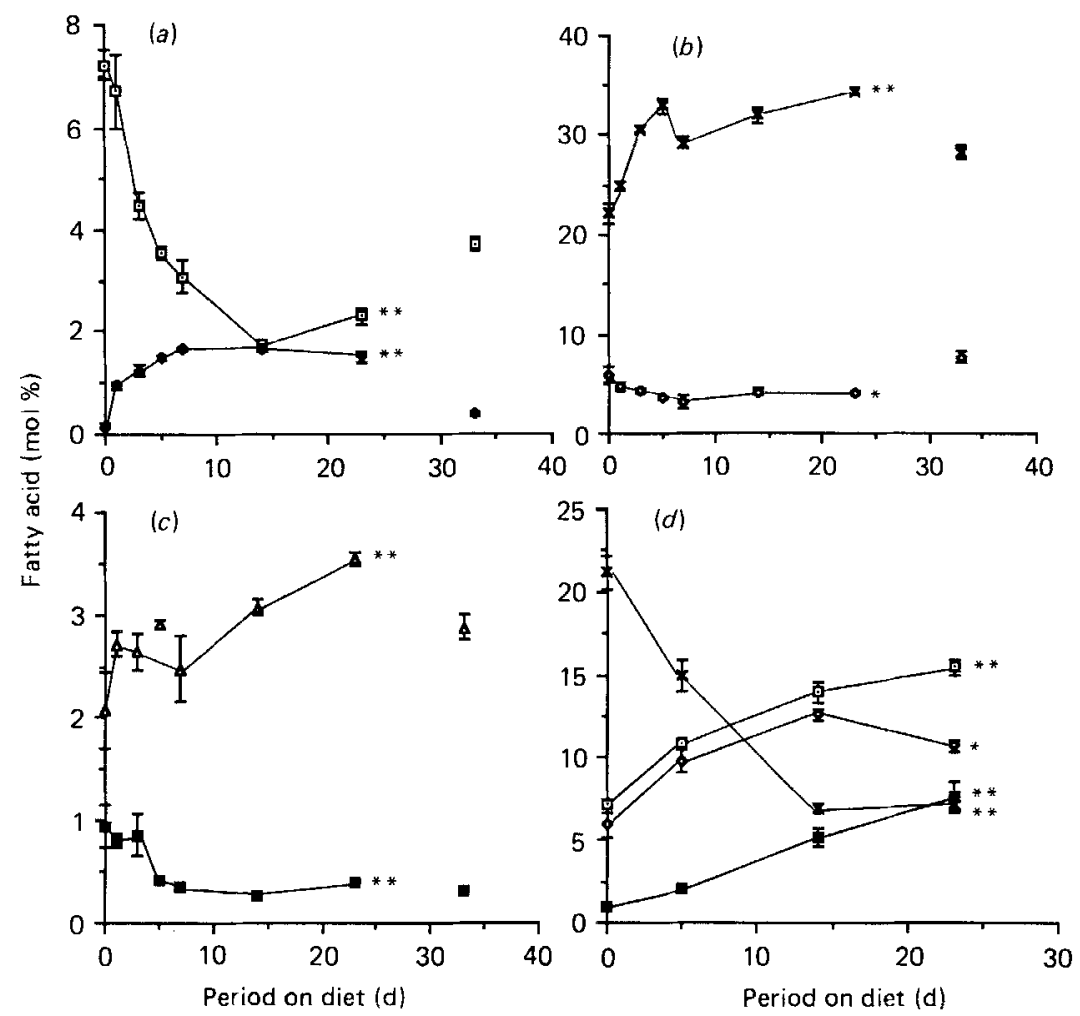

Fig. 1. Incorporation of selected $n-6$ and $n-3$ polyunsaturated fatty acids into heart choline phospholipids isolated from mice given menhaden oil (MO) or hydrogenated coconut oil (HCO) for $23 \mathrm{~d}$. After $23 \mathrm{~d}$, mice receiving MO were given the HCO diet for an additional $10 \mathrm{~d}$ (unconnected symbols). (a) MO, ( $\square$ )

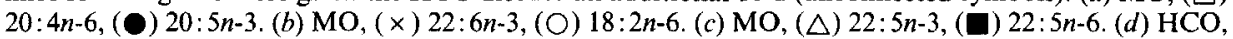

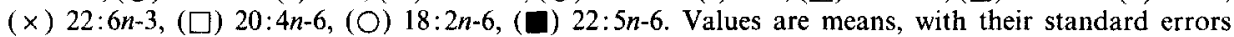
represented by vertical bars $(n 4)$. Differences in fatty acid mean values between days 0 and 23 that were statistically significant are represented by: $* P<0 \cdot 05,{ }^{* *} P<0.01$.

\section{Extraction and analysis of lipids}

Mice were killed by cervical dislocation. The heart was removed as described previously (Swanson et al. 1987a). The cranium was opened with an incision at the base of the head and the brain tissue removed. Both organs were washed in ice-cold isotonic saline $(9 \mathrm{~g}$ sodium chloride/1), blotted on paper to remove excess fluid, weighed, frozen in liquid $\mathrm{N}_{2}$ and stored at $-70^{\circ}$ until analysed.

Tissue lipids were extracted and individual PL classes separated and saponified as reported by Swanson et al. (1987a). Free fatty acids were then dissolved in $200 \mu 1$ diethyl ether and converted into fatty acid methyl esters (FAME) using $100 \mu 1$ diazomethane at room temperature for $15 \mathrm{~min}$. FAME were separated and quantified by gas-liquid chromatography (5880A gas-liquid chromatograph; Hewlett Packard, Avondale, PA) using a $60 \mathrm{~m} \times 0.75 \mathrm{~mm}$ SP-2330 glass capillary column (Supelco, Bellefonte, PA). Flowrate was set at $2.5 \mathrm{ml} / \mathrm{min}$. The oven temperature was programmed at $5 \% / \mathrm{min}$ from 140 to $240^{\circ}$. FAME were identified by comparison of retention times with standards prepared from shark liver oil (Bruckner et al. 1984). 

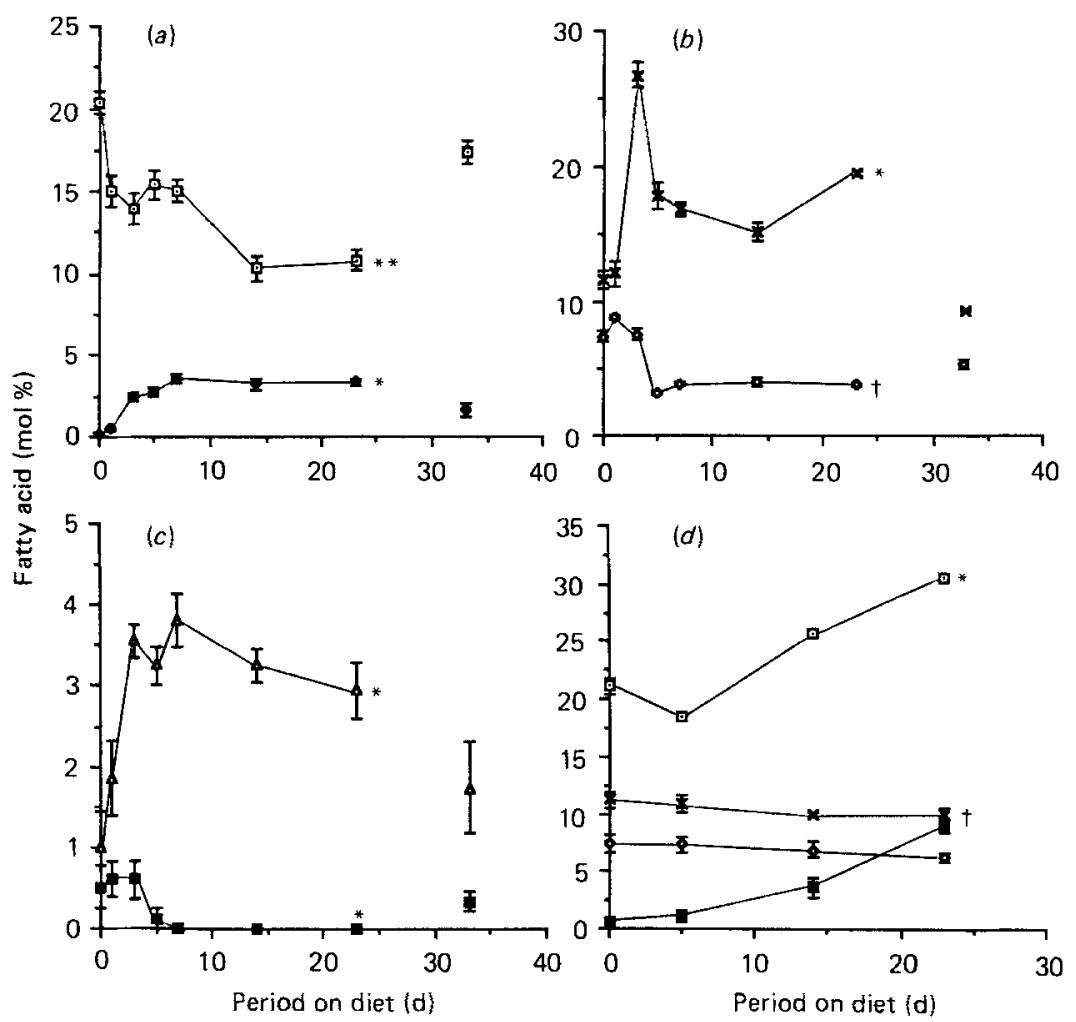

Fig. 2. Incorporation of selected $n-6$ and $n-3$ polyunsaturated fatty acids into heart serine-inositol phospholipids isolated from mice given menhaden oil (MO) or hydrogenated coconut oil (HCO) for $23 \mathrm{~d}$. After $23 \mathrm{~d}$, mice receiving MO were given the $\mathrm{HCO}$ diet for an additional $10 \mathrm{~d}$ (unconnected symbols). (a) MO, ( $\square$ ) 20:4n-6, (О) 20:5n-3. (b) MO, (× ) 22:6n-3, (O) 18:2n-6. (c) MO, ( $\triangle$ ) 22:5n-3,

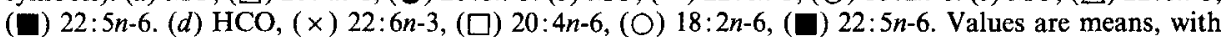
their standard errors represented by vertical bars $(n 4)$. Differences in fatty acid mean values between days 0 and 23 that were statistically significant are represented by: $\nmid P<0 \cdot 10,{ }^{*} P<0.05,{ }^{* *} P<0.01$.

\section{Statistical analysis}

Statistical significance of mean differences between dietary treatments and within each dietary treatment were determined by analysis of variance (Goodnight, 1979).

\section{RESULTS}

The changes in the contents of saturated and monounsaturated fatty acids were minor (values not shown) compared with the alterations observed in individual PL-PUFA composition. Therefore, only values relating to modification of specific $n-6$ and $n-3$ PUFA in cardiac and brain PL classes are presented.

\section{Heart lipids}

Rates of incorporation. The most significant changes in $n$-3 PUFA composition occurred during the first week of MO consumption in each PL class (Figs. 1-3 $(a-c)$ ). A rapid rate of incorporation of $20: 5 n-3,22: 5 n-3$ and $22: 6 n-3$ was observed. For example, the content of 20:5n-3 increased immediately following $1 \mathrm{~d}$ of $\mathrm{MO}$ ingestion and continued to increase 

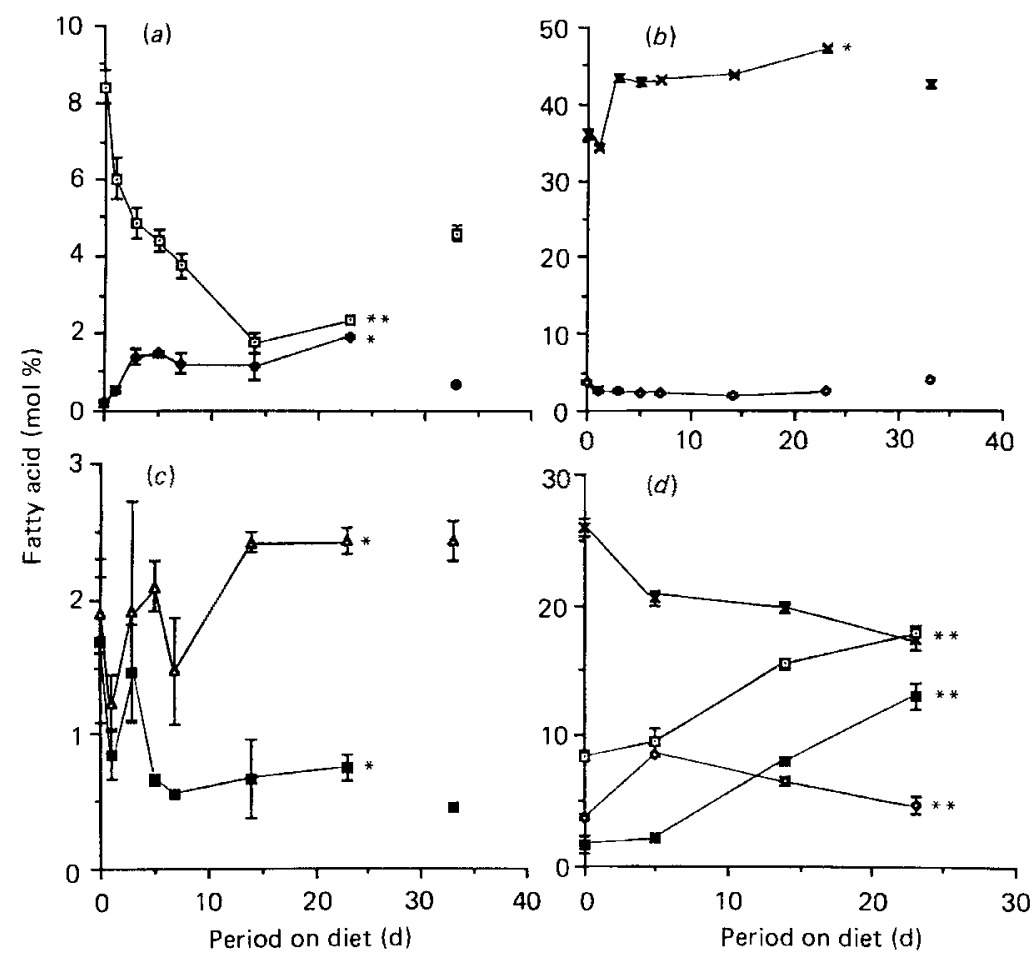

Fig. 3. Incorporation of selected $n-6$ and $n-3$ polyunsaturated fatty acids into heart ethanolamine phospholipids isolated from mice given menhaden oil (MO) or hydrogenated coconut oil (HCO) for $23 \mathrm{~d}$. After $23 \mathrm{~d}$, mice receiving MO were given the $\mathrm{HCO}$ diet for an additional $10 \mathrm{~d}$ (unconnected

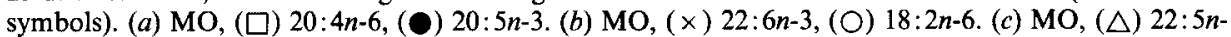

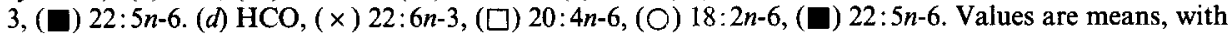
their standard errors represented by vertical bars $(n 4)$. Differences in fatty acid mean values between days 0 and 23 that were statistically significant are represented by: ${ }^{*} P<0 \cdot 05,{ }^{* *} P<0 \cdot 01$.

steadily for 1 week (Figs. 1-3(a)). The content of 22:6n-3 increased rapidly between days 0 and $3 ; 9 \mathrm{~mol} \%$ in choline PL (PC), $15 \mathrm{~mol} \%$ in serine-inosital PL (PS/PI), and $7 \mathrm{~mol}$ $\%$ in ethanolamine PL (PE). After day 3 , the increase in $n$ - 3 PUFA was more gradual (Figs. $1-3(b))$.

The increase in $n-3$ PUFA occurred with a concomitant decrease in the content of $n-6$ PUFA (Figs. 1-3(a-c)). The rate of decrease in the $n-6$ PUFA $(18: 2 n-6,20: 4 n-6$ and $22: 5 n$ 6) varied between PL class. A marked decrease in the content of $20: 4 n-6$ was observed immediately following $1 \mathrm{~d}$ of MO ingestion in cardiac PS/PI and PE (Figs. 2 and 3(a)) and after day 3 in PC (Fig. 1(a)). After day 1, further reductions in the content of $20: 4 n-6$ were observed at a very steady rate in cardiac PC and PE (Figs. 1 and $3(a)$ ). The content of $18: 2 n-6$ also decreased at a steady rate, while the level of $22: 5 n-6$ was held constant in cardiac PC, PS/PI and PE until day 3 when a marked and rapid decrease was observed in all PL classes (Figs. 1-3( $b$ and $c)$ ).

Time-period to reach steady-state. We define the time of steady-state as the point when no further changes in the PUFA composition were observed in tissue PL classes with continued MO consumption. This time-point was variable for each PUFA within each PL class. The steady-state time-point for $20: 5 n-3$ was reached by day 7 for cardiac PC, PS/PI and by day 3 for PE (Figs. 1-3(a)); for $22: 6 n-3$, it was observed by day 3 for PC and PE 

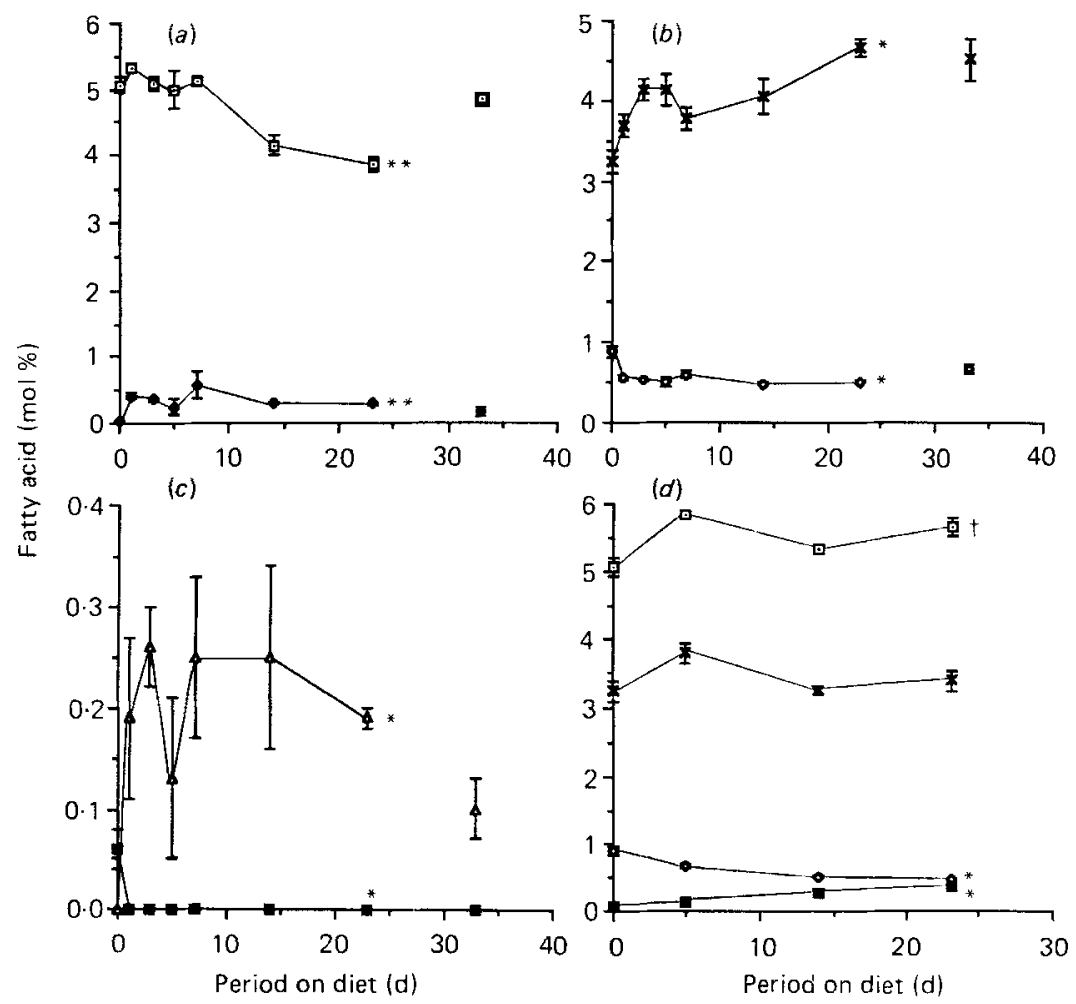

Fig. 4. Incorporation of selected $n-6$ and $n-3$ polyunsaturated fatty acids into brain choline phospholipids isolated from mice given menhaden oil (MO) or hydrogenated coconut oil ( $\mathrm{HCO}$ ) for $23 \mathrm{~d}$. After $23 \mathrm{~d}$, mice receiving MO were given the $\mathbf{H C O}$ diet for an additional $10 \mathrm{~d}$ (unconnected symbols). (a) MO, ( $\square$ )

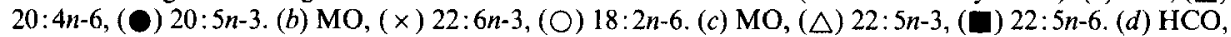
(×) $22: 6 n-3$, (ロ) $20: 4 n-6$, (O) $18: 2 n-6$, (ロ) $22: 5 n-6$. Values are means, with their standard errors represented by vertical bars $(n 4)$. Differences in fatty acid mean values between days 0 and 23 that were statistically significant are represented by: $\nmid P<0 \cdot 10,{ }^{*} P<0 \cdot 05,{ }^{* *} P<0 \cdot 01$.

(Figs. 1 and 3(b)). However, a small further increase was observed between days 14 and 23 in both PC and PE and a second steady-state was not reached before the termination of the MO supplementation period. The findings show that $22: 6 n-3$ was preferentially incorporated into heart PL compared with $20: 5 n-3$. For example, the content of $22: 6 n-3$ increased by 11.3 and $10.7 \mathrm{~mol} \%$ while $20: 5 n-3$ increased by only 1.6 and $1.7 \mathrm{~mol} \%$ in PC and $\mathrm{PE}$ respectively during the $23 \mathrm{~d}$ MO supplementation period. The enhanced incorporation of $22: 6 n-3$ relative to $20: 5 n-3$ was observed even though the content of $20: 5 n-3$ in the MO diet was three times greater than that of 22:6n-3 (Table 1).

Rate of n-3 PUFA depletion. The increased content of $n-3$ PUFA was maintained or increased slightly $(22: 6 n-3)$ with continued MO consumption in cardiac PL. However, the content of $n-3$ PUFA decreased rapidly following cessation of dietary MO (Figs. 1-3 $(a-c)$ ). The average reduction of $20: 5 n-3$ and $22: 6 n-3$ was 58 and $29 \%$ respectively of the maximum level in cardiac PL. The $10 \mathrm{~d}$ depletion trial was of sufficient length to allow repletion of $18: 2 n-6$ to initial levels. However, the contents of $20: 4 n-6$ and $22: 5 n-6$ were only partly repleted within this period (Figs. 1-3(a-c)).

Time-effects on cardiac PUFA composition. The HCO dietary group allowed us to compare changes in PUFA composition with time between an $n-3$ PUFA-rich diet (MO) and 

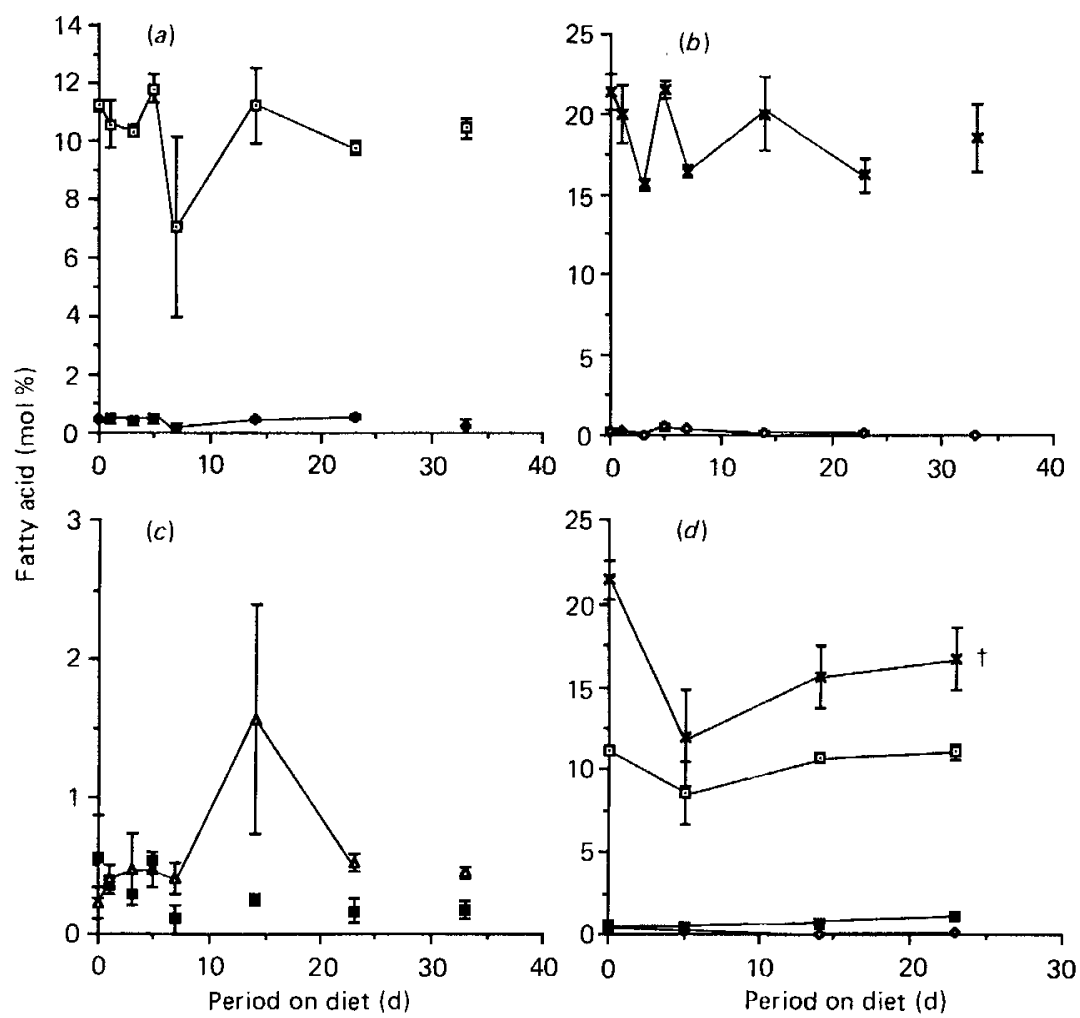

Fig. 5. Incorporation of selected $n-6$ and $n-3$ polyunsaturated fatty acids into brain serine-inositol phospholipids isolated from mice given menhaden oil (MO) or hydrogenated coconut oil (HCO) for $23 \mathrm{~d}$. After $23 \mathrm{~d}$, mice receiving $\mathrm{MO}$ were given the $\mathrm{HCO}$ diet for an additional $10 \mathrm{~d}$ (unconnected

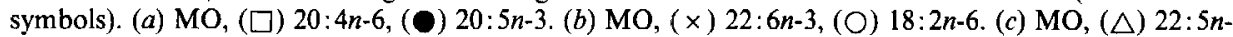

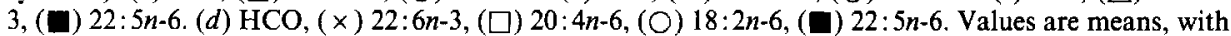
their standard errors represented by vertical bars. Differences in fatty acid mean values between days 0 and 23 that were statistically significant are represented by: $\dagger P<0 \cdot 10$.

an $n$-3 PUFA-deficient diet (HCO). Mice given the $\mathrm{HCO}$ diet were capable of incorporating $n$-6 PUFA and elongating/desaturating $18: 2 n-6$ to $20: 4 n-6$ and $22: 5 n-6$ very efficiently (Figs. 1-3(d)). In contrast, the content of $22: 6 n-3$ decreased with time. These results indicate that the changes observed in mice given the MO diet were not caused by any time-related factors. Therefore, the observed PUFA modifications in cardiac PL classes were principally influenced by the dietary oil provided.

\section{Brain lipids}

Rate of incorporation. The kinetics of the incorporation of $n-3$ PUFA into brain PL classes were quite different compared with the heart. 'There was an immediate increase in the content of 20:5n-3 in all PL classes after $1 \mathrm{~d}$ of MO supplementation (Figs. 4-6(a)). In PE, the content of $20: 5 n-3$ continued to increase until day 5 (Fig. 6(a)). The content of 22:6n3 in PC increased immediately and steadily until day 5; in PE no change in 22:6n-3 was observed until day 7 and in PS/PI the content of $22: 6 n-3$ fluctuated widely throughout the study (Figs. 4-6(b)).

As in cardiac PL, a concurrent reduction of $n-6$ PUFA (18:2n-6, 20:4n-6 and $22: 5 n-6)$ occurred as the $n-3$ PUFA content increased (Figs. 4-6 (a-c)). However, in contrast to the 

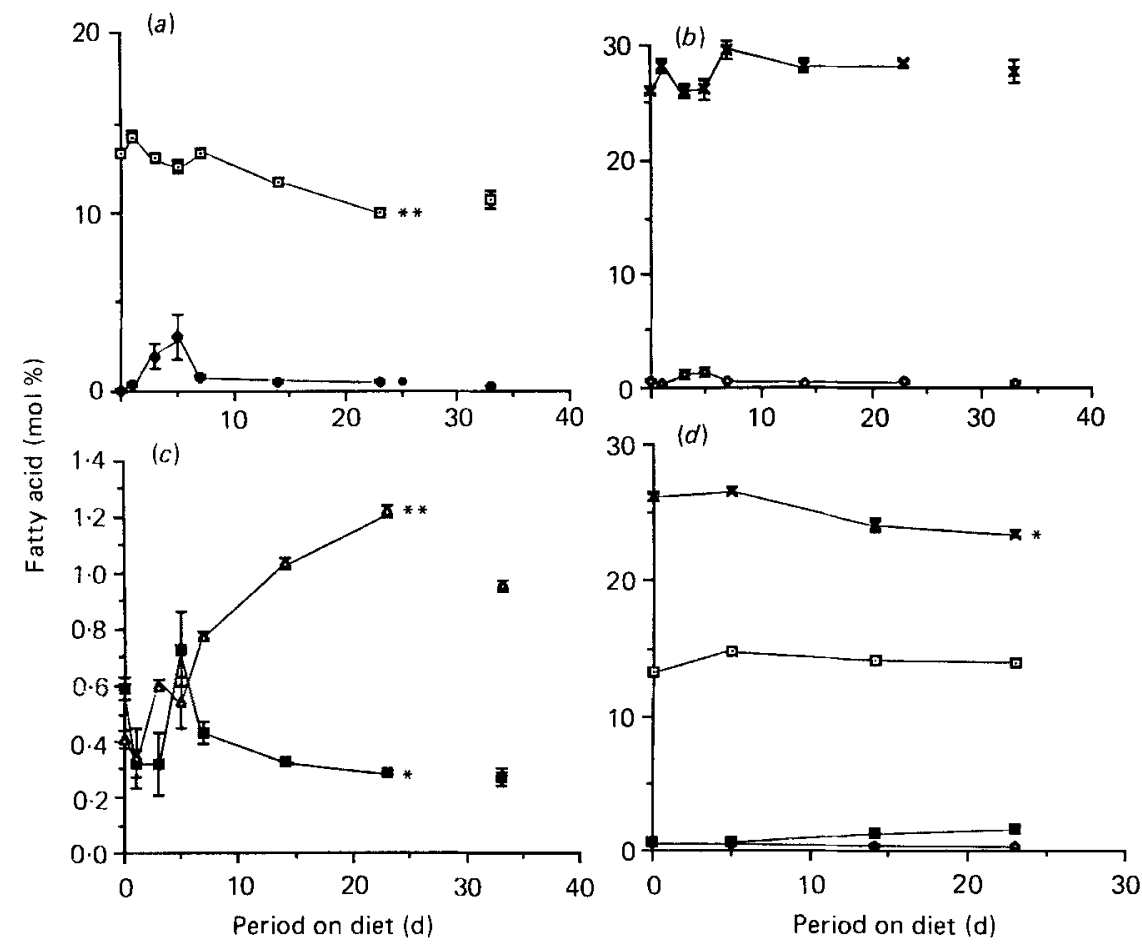

Fig. 6. Incorporation of selected $n-6$ and $n-3$ polyunsaturated fatty acids into brain ethanolamine phospholipids isolated from mice given menhaden oil (MO) or hydrogenated coconut oil (HCO) for $23 \mathrm{~d}$. After $23 \mathrm{~d}$, mice receiving $\mathrm{MO}$ were given the $\mathrm{HCO}$ diet for an additional $10 \mathrm{~d}$ (unconnected

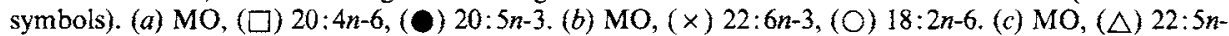

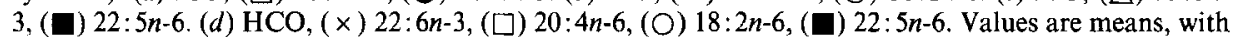
their standard errors represented by vertical bars $(n 4)$. Differences in fatty acid mean values between days 0 and 23 that were statistically significant are represented by: ${ }^{*} P<0.05,{ }^{* *} P<0.01$.

immediate and steady rate of reduction of $20: 4 n-6$ observed in cardiac PL, a lag period of 5-7 d when the content of 20:4n-6 did not change was observed (Figs. 4-6 (a)). After this lag period, a marked decrease in the content of 20:4n-6 ensued (Figs. 4-6(a)). The content of $18: 2 n-6$ fluctuated in both PE and PS/PI during MO supplementation while in PC the content decreased immediately after $1 \mathrm{~d}$ (Figs. 4-6 (b)). An immediate reduction in the content of $22: 5 n-6$ was also observed in each PL class by day 1 (Figs. 4-6(c)).

Time-period to reach steady-state. The time-period required to reach steady-state varied between fatty acids and PL classes (Figs. 4 6(a-c)). For example, the content of $20: 5 n-3$ in PC reached a steady-state after day 1 , in PE this state was not attained until day 7 and in PS/PI the content of 20:5n-3 did not change during the MO supplementation period (Figs. 4-6(a)). The content of 22:6n-3 reached a steady-state in PE by day 7 (Fig. 6(b)). However, in PC the content of this PUFA was still increasing when MO supplementation was discontinued (Fig. 4(b)).

In contrast to the heart, the content of $20: 4 n-6$ in brain $\mathrm{PL}$ did not reach a new reduced steady-state during the 3-week feeding trial (Figs. 4-6(a)). In brain PC and PE, the content of 20:4n-6 was still decreasing when MO supplementation was discontinued. In PC, the content of $18: 2 n-6$ reached a reduced steady-state by day 1 ; however, no significant changes were observed in the content of $18: 2 n-6$ in PE or PS/PI (Figs. 4-6(b)). 
Rate of $\mathrm{n}-3$ PUFA depletion. The MO-induced PUFA modifications were not as readily reversed as those observed in the heart. During the $10 \mathrm{~d} n-3$ depletion period, the content of $20: 4 n-6$ increased by only $1 \mathrm{~mol} \%$ in PC, PE and PS/PI with a concomitant reduction in the content of 22:5n-3 (Figs. 4-6(a-c)). However, the content of both 20:5n-3 and $22: 6 n-3$ in brain PC and PE did not change markedly during this depletion period (Figs. 4 and $6(a, b)$ ). This lack of an immediate change in brain PL PUFA composition during the $n$-3 PUFA depletion period is consistent with the lag period we observed for 20:4n-6 and 22:6n-3 in selected brain PL classes during the supplementation period.

Time-effects on brain PUFA composition. The effects of the $n$-3-deficient diet $(\mathrm{HCO})$ on mouse brain PL PUFA composition were similar to those observed for the heart (Figs. $1-6(d)$ ). The content of $n-6$ PUFA either increased or did not change while the content of 22: $6 n-3$ decreased or remained unchanged in brain PL classes (Figs. 4-6(d)). Although the magnitude of incorporation of $n-3$ PUFA was small, the elevated levels in mice given MO were consistently greater than in mice given HCO. Moreover, the content of $n-6$ PUFA was greater in mice receiving $\mathrm{HCO}$ relative to mice given $\mathrm{MO}$, which indicates the sensitivity of brain PUFA composition to dietary lipid (Figs.4-6(a-d)).

\section{DISCUSSION}

A differential rate and extent of incorporation and depletion of $n-3$ PUFA in tissue phospholipids has been shown in this time-course study. The greatest change in heart and brain PL PUFA composition consistently occurred between days 0 and 7 . Furthermore, the PUFA modifications induced by dietry MO were reversible when MO was discontinued. These observations are generally consistent with other time-course studies which show modification of the fatty acid composition of plasma lipid components in human beings and animals following consumption of fish oil (Thorngren \& Gustafson, 1981; Terano et al. 1983; von Schacky et al. 1985; von Schacky \& Weber, 1985). Iritani \& Narita (1984) have also reported comparable changes in liver lipids from essential-fatty-acid-deficient rats given fish oil.

The magnitude of change in phospholipid $n$-3 PUFA composition is not strictly proportional to the level of fish oil $n-3$ PUFA in the diet (Sanders \& Roshanai, 1983; Swanson \& Kinsella, 1986) nor is it directly related to the duration of fish oil consumption (von Schacky \& Weber, 1985; Knapp et al. 1986; Swanson et al. 1987a). In the present study, the maximum reduction of $n$ - 6 PUFA and maximum increase in $n-3$ PUFA contents occurred within 2 weeks. The lack of a linear relation between dose and duration of fish oil consumption may be related to the attainment of a steady-state between dietary PUFA and endogenous PUFA. In turn, this effect may reflect an alteration in the affinity or specific activity, or both, of fatty acid acylases, transferases and desaturases. In addition, it may be associated with the existence of different PUFA pools varying in turnover times. The time-period required to reach this steady-state, as shown in the present study and others, varies between tissue and lipid classes (Gudbjarnason et al. 1978; Thorngren \& Gustafson, 1981; Iritani \& Narita, 1984; Swanson et al. 1987a). This inconsistency makes it difficult to predict the rate and magnitude of the effects of dietary fish oil on many relevant physiological processes such as lipid and eicosanoid synthesis, activity of desaturases and membrane-bound enzymes, inflammatory responses and vascular functions.

Currently, an accurate estimation of the time-period required to reach steady-state for different tissues has not been established. Results from several human studies which have used high doses of fish oil supplementation $(10-40 \mathrm{ml} / \mathrm{d})$, suggest that the length of time is a matter of weeks rather than days (von Schacky et al. 1985; von Schacky \& Weber, 
1985). The findings from these studies are consistent with our results which show that on average the time-period to reach steady-state PUFA status is between 1 and 2 weeks in organs from mice given MO.

The ability of dietary fish oils to modify the PUFA composition of organ PL is important, since membrane PL are the main source of PUFA for eicosanoid synthesis. Differences in the rate, magnitude and reversibility of $n$-3 PUFA incorporation between organ and PL class may also indicate differential effects on the capacity of an organ to produce eicosanoids when stimulated. Knapp et al. (1986) observed a significant decrease in urinary excretion of TXA $_{2}$ metabolites by patients with atherosclerosis, after 1 week of consumption of $50 \mathrm{ml}$ fish oil/d. In these same volunteers, a reduced steady-state for $\mathrm{TXA}_{2}$ excretion was observed between weeks 1 and 3 . These changes in TXA $\mathbf{A}_{2}$ synthesis occurred in conjunction with an increased incorporation of $20: 5 n-3$ and $22: 6 n-3$ and a reduction in the content of 20:4n-6 in erythrocyte PL. Although the time-course for the modification of erythrocyte PL PUFA composition was not reported, the greatest change in PUFA composition was noted during the first week of supplementation, which agrees with our findings.

The similarities between the pattern of change in organ PUFA composition reported in the present study relative to the change in $\mathrm{TXA}_{2}$ concentration reported by Knapp et al.

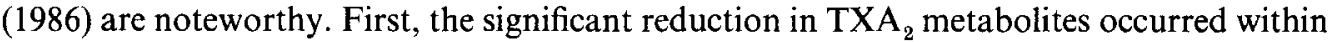
1 week of supplementation, which corresponds to the period of greatest change in organ PUFA composition. Second, steady-state for reduced $\mathrm{TXA}_{2}$ synthesis was reached at approximately 1 or 2 weeks after the time of steady-state for organ PUFA composition. Therefore, it appears that the change in eicosanoid synthesis closely reflects the changes induced by dietry fish oil in tissue fatty acid composition.

This time-course study and one reported previously (Swanson et al. 1987a) monitored the modifications of PUFA composition of four different organs (brain, heart, lung and kidney) from mice given dietary MO for 3 weeks. Differences between tissues were found in the magnitude of incorporation of $n-3$ PUFA and reductions in $n-6$ PUFA, in addition to differences in rates of incorporation and depletion of these PUFA. For example, the magnitude of incorporation of $20: 5 n-3$ in total PL of the lung and kidney was 14.06 and $27.91 \mathrm{~mol} \%$ respectively, whereas in the heart and brain the increase was only 6.38 and $0.85 \mathrm{~mol} \%$ respectively, for the 3 week MO supplementation period. In addition, in the lung and kidney the mol \% of 20:5n-3 exceeded that of $20: 4 n-6$ in PC and PE. However, in heart and brain PL the mol \% of $20: 5 n-3$ never exceeded that of $20: 4 n-6$.

In contrast, we observed a preferential incorporation of $22: 6 n-3$ relative to $20: 5 n-3$ in PL of mouse brain and heart. Differences were also observed with respect to rates of depletion of $n-3$ PUFA following cessation of MO supplementation. For example, the replacement of 20:5n-3 by $n-6$ PUFA was greater in the lung and kidney (the two organs which preferentially incorporated $20: 5 n-3$ ), while in the heart and brain (the two organs which preferentially incorporated $22: 6 n-3)$ the mol $\%$ of $22: 6 n-3$ was reduced to a greater extent than $20: 5 n-3$ by $n-6$ PUFA. von Schacky et al. (1985) also noted a similar tendency of erythrocyte lipids, which preferentially incorporated $20: 5 n-3$, to retain $22: 6 n-3$ longer than $20: 5 n-3$. These results suggest that PUFA are not only preferentially incorporated into organ PL, but are also selectively depleted.

The results of the present study suggest that the duration of consumption of moderate doses of dietary fish oil must be of at least 1 week and be maintained for intervals much shorter than $10 \mathrm{~d}$ to maintain elevated $n$-3 PUFA levels in heart and brain PL. The differential responses of organs and PL classes to MO supplementation also indicate that differences in physiological responses known to be affected by fish oils may also occur at different rates and magnitudes. Further research is required to ascertain the relation between the kinetics of $n$-3 PUFA incorporation and physiological processes. 
The authors wish to thank Drs B. R. Lokesh and J. B. German for their advice and technical assistance. This project was funded in part by the New York SeaGrant Foundation and USDA Nutrition Grant no 86-CRCR-1-2186.

\section{REFEREIJCES}

Bruckner, G. G., Lokesh, B. R., German, B. \& Kinsella, J. E. (1984). Thrombosis Research 34, 479-497.

Buege, J. A. \& Aust, S. D. (1978). Methods in Enzymology 52, 306.

Croft, K. D., Beilin, C. J., Vandongen, R. \& Mathews, E. (1984). Biochimica et Biophysica Acta 795, $196-207$.

Flier, J., Lokesh, B. R. \& Kinsella, J. E. (1985). Nutrition Research 5, 277-283.

Goodnight, J. H. (1979). In Statistical Analysis Systems, SAS Users Guide, pp. 113-229 [J. T. Helwig and R. A. Council, editors]. Raleigh, NC: SAS Institute Inc.

Gudbjarnason, S., Oskarsdottir, G., Doell, B. \& Hallgrimsson, J. (1978). Advances in Cardiology 25, $130-144$.

Herold, P. M. \& Kinsella, J. E. (1986). American Journal of Clinical Nutrition 43, 566-598.

Iritani, N. \& Narita, R. (1984). Biochimica et Biophysica Acta 793, 441-447.

Knapp, H. R., Reilly, I. A. G., Alessandrini, P. \& Fitzgerald, G. A. (1986). New England Journal of Medicine 314, 937-942.

Lands, W. E. M. (1986). Fish and Human Health. New York : Academic Press.

Lokesh, B. R., Bruckner, G. G. \& Kinsella, J. E. (1985). Lipids 20, 842-849.

Sanders, T. A. B. (1985a) In The Role of Fats in Human Nutrition, pp. 101-116. [F. B. Padley and J. Podmore, editors]. Chichester : Ellis Horwood.

Sanders, T. A. B. (1985 b). Proceedings of the Nutrition Society 44, 391-397.

Sanders, T. A. B. \& Roshanai, F. (1983). Clinical Science 64, 91-99.

Simopoulos, A. P., Kifer, R. R. \& Martin, R. E. (1986). Health Effects of Polyunsaturated Fatty Acids in Seafoods. New York: Academic Press, Inc.

Swanson, J. E., Black, J. M. \& Kinsella, J. E. (1987a). Journal of Nutrition 117, 824-832.

Swanson, J. E. \& Kinsella, J. E. (1986). Journal of Nutrition 116, 514-523.

Swanson, J. E., Lokesh, B. R. \& Kinsella, J. E. (1987b). Federation Proceedings 46, 1170 (abstract).

Terano, T., Hirai, A., Hamazaki, T., Kobayashi, S., Fujita, T., Tamara, Y. \& Kumagai, A. (1983). Atherosclerosis 46, 321-331.

Thorngren, M. \& Gustafson, A. (1981). Lancet ii, 1190-1193.

von Schacky, C., Fisher, S. \& Weber, P. C. (1985). Journal of Clinical Investigation 76, 1626-1631.

von Schacky, C. \& Weber, P. C. (1985). Journal of Clinical Investigation 76, 2446-2450. 